\title{
LETTER \\ Single Image Dehazing Using Invariance Principle
}

\author{
Mingye $\mathrm{JU}^{\dagger}$, Zhenfei GU ${ }^{\dagger}$, Dengyin ZHANG ${ }^{\dagger a)}$, Nonmembers, and Jian LIU ${ }^{\dagger \dagger}$, Student Member
}

SUMMARY In this letter, we propose a novel technique to increase the visibility of the hazy image. Benefiting from the atmospheric scattering model and the invariance principle for scene structure, we formulate structure constraint equations that derive from two simulated inputs by performing gamma correction on the input image. Relying on the inherent boundary constraint of the scattering function, the expected scene albedo can be well restored via these constraint equations. Extensive experimental results verify the power of the proposed dehazing technique.

key words: image dehazing, inhomogeneous atmosphere, atmospheric scattering model, depth map

\section{Introduction}

Images of outdoor scenes are often degraded by the atmospheric suspended particles, thus removing such negative visual effects has strong implications in many computer vision systems. Currently, these existing approaches for haze removal can be grouped into the contrast-based methods [1][3], the fusion-based methods [4], [5] and the physicallybased methods [6]-[22].

Generally, the physically-based methods are able to achieve better performance than others since they take the degradation mechanism [6] into consideration and restore haze-free image via strong prior knowledge. However, the inner limitations still exist in some particular cases. For instance, Tan [7] estimated the transmission by maximizing the contrast within a local patch based on Markov Random Field, but this approach tends to yield an over-saturated result. Nishino [8] adopted the Bayesian posterior probability model to remove haze, in which the latent statistical structure was fully leveraged. Although this method can achieve impressive results for dense hazy images, it may produce over-enhanced output in case of mist. Fattal [9] assumed that the transmission and surface shading are locally uncorrelated, and removes haze based on independent component analysis (ICA), but it may be invalid when hazy image lacks sufficient color information and cannot be used for grayscale image. Tarel [10] estimated the atmospheric

Manuscript received June 18, 2017.

Manuscript revised August 9, 2017.

Manuscript publicized September 1, 2017.

$\dagger$ The authors are with School of Internet of Things, Nanjing University of Posts and Telecommunications, Xinmofan Road \#66, Nanjing, 210003, China.

${ }^{\dagger}$ The author is with College of Communication and Information Engineering, Nanjing University of Posts and Telecommunications, Xinmofan Road \#66, Nanjing, 210003, China.

a) E-mail: Zhangdy@njupt.edu.cn (Corresponding author)

DOI: 10.1587/transinf.2017EDL8134 veil using the median filter. Owing to the poor performance of the median filter for edge preserving, a small amount of mist would remain around the depth abrupt. He [11] discovered the dark channel prior for haze removal, however, this prior may not work for the region where the scene brightness is inherently similar to the atmospheric light. Zhu [12] created a linear model for the scene depth using the color attenuation prior, and this allows us to obtain the depth map directly. However, this method is subject to the weak robustness because the scattering coefficient estimation method is not given. Recently, other studies [13]-[15] combine hazerelevant features with learning frameworks to estimate the transmission, and their methods give a new strategy for image dehazing, but the dehazing ability is obviously weaker than others due to the insufficient training samples.

In this letter, we present a novel single image haze removal technique based on invariance principle. Our technique does not utilize any image priors; instead, we simulate two inputs under different weather conditions from an original hazy image using gamma correction, and formulate structure constraint equations for these two simulated inputs on the basis of the invariance principle and the atmospheric scattering model (ASM). Using these constraint equations, and combined with the inherent boundary constraint on the scattering function, we can accurately recover the expected scene albedo. Experimental results demonstrate that the proposed technique outperforms several relevant state-of-theart methods in terms of both robustness and effectiveness.

\section{The Proposed Technique}

In computer vision and computer graphics, the atmospheric scattering model [6], [16]-[18] is widely used to describe the degradation mechanism of a hazy image, which is expressed as

$$
I^{c}(x, y)=A^{c} \cdot \rho^{c}(x, y) \cdot e^{-\beta \cdot d(x, y)}+A^{c} \cdot\left(1-e^{-\beta \cdot d(x, y)}\right)
$$

where $\mathrm{c} \in\{\mathrm{r}, \mathrm{g}, \mathrm{b}\}$ is color channel index, $I^{c}$ and $\rho^{c}$ are the hazy image and scene albedo, respectively, $A^{c}$ is the global atmospheric light, $d$ is the scene depth, $\beta$ is the scattering coefficient and assumed to be constant in [6], [12]. It is obvious that the single image dehazing is an ill-posed problem, because the available scene structure information is significantly insufficient. In spite of this, the scene albedo and scene depth for a determined nature image are both invariant even after manual post-processing, and we name this statistical regularity as invariance principle. This inspires us to 
present a new technique for single image dehazing. The fundamental idea behind the proposed technique is to extract some important information of different weather conditions, by constructing constraint equations on two hazy images. Obviously, the method of simulating hazy images from the original input is the key to effectively remove the haze.

Liu et al. [19] employ the gamma correction to preprocess the input hazy image and achieve a pleasing restoration result based on ASM, which may imply that the preprocessing results are very consistent with real nature scenes. Inherently inspired by this, we first attempt to generate two inputs taken in different weather conditions by performing the gamma correction on the input hazy image

$$
\left\{\begin{array}{l}
\tilde{I}^{c}(x, y)=\left(I^{c}(x, y)\right)^{\tilde{\gamma}} \\
\check{I}^{c}(x, y)=\left(I^{c}(x, y)\right)^{\check{\gamma}}
\end{array}\right.
$$

where gamma correction factors $\tilde{\gamma}<1$ and $\check{\gamma}>1$ are used to generate the denser image and relatively misty one, respectively. If the simulated images $\tilde{I}^{c}$ and $\check{I}^{c}$ are subject to the ASM, by means of the invariance principle, the Eq. (2) can be transformed into

$$
\left\{\begin{array}{c}
\tilde{I}^{c}(x, y)=\tilde{A}^{c}\left(\rho^{\mathrm{c}}(x, y) \cdot e^{-\tilde{\beta} \cdot d(x, y)}+\left(1-e^{-\tilde{\beta} \cdot d(x, y)}\right)\right) \\
=\left(A^{\mathrm{c}}\right)^{\tilde{\gamma}} \cdot\left\{\left(\rho^{\mathrm{c}}(x, y)-1\right) \cdot e^{-\beta \cdot d(x, y)}+1\right\}^{\tilde{\gamma}} \\
\check{I}^{c}(x, y)=\check{A}^{c}\left(\rho^{c}(x, y) \cdot e^{-\check{\beta} \cdot d(x, y)}+\left(1-e^{-\check{\beta} \cdot d(x, y)}\right)\right) \\
=\left(A^{\mathrm{c}}\right)^{\check{\gamma}} \cdot\left\{\left(\rho^{\mathrm{c}}(x, y)-1\right) \cdot e^{-\beta \cdot d(x, y)}+1\right\}^{\check{\gamma}}
\end{array}\right.
$$

where $\tilde{A}^{c}, \tilde{\beta}$ and $\check{A}^{c}, \check{\beta}$ are the atmospheric light and scattering coefficient with respect to $\tilde{I}^{c}$ and $\check{I}^{c}$, respectively. Simplifying the Eq. (3), and let $\tilde{A}^{c}=\left(A^{\mathrm{c}}\right)^{\tilde{\gamma}}, \check{A}^{c}=\left(A^{\mathrm{c}}\right)^{\check{\gamma}}$ yields

$$
\left\{\begin{array}{c}
\left(\rho^{\mathrm{c}}(x, y)-1\right) \cdot e^{-\tilde{\beta} \cdot d(x, y)} \\
=\left\{\left(\rho^{\mathrm{c}}(x, y)-1\right) \cdot e^{-\beta \cdot d(x, y)}+1\right\}^{\tilde{\gamma}}-1 \\
\left(\rho^{\mathrm{c}}(x, y)-1\right) \cdot e^{-\breve{\beta} \cdot d(x, y)} \\
=\left\{\left(\rho^{\mathrm{c}}(x, y)-1\right) \cdot e^{-\beta \cdot d(x, y)}+1\right\}^{\check{\gamma}}-1
\end{array}\right.
$$

Since the $(\rho(x, y)-1) \cdot \exp \{-\beta \cdot d(x, y)\}$ is relatively close to 0 in general, especially for dense hazy regions. Thus, according to equivalent infinitesimal theorem, the Eq. (4) is equivalent to

$$
\left\{\begin{array}{l}
\tilde{\beta} \cong \beta-\frac{\ln (\tilde{\gamma})}{d} \\
\check{\beta} \cong \beta-\frac{\ln (\check{\gamma})}{d}
\end{array}\right.
$$

The Eq. (5) concludes that we can adjust the concentration of haze by changing gamma correction factor, but the simulated results should be regarded as inhomogeneous hazy images.

Based on the above conclusion, we redefine the scattering coefficients $\tilde{\beta}$ and $\breve{\beta}$ as the scattering maps $\tilde{\beta}^{\prime}(x, y)$ and $\check{\beta}^{\prime}(x, y)$, which vary along with the pixel spatial location.

According to Eq. (3) and the redefined scattering maps, the scene albedo can be computed as

$$
\rho^{c}(x, y)=1+\frac{\tilde{I}^{c}(x, y)-\tilde{A}^{c}}{\tilde{A}^{c} \cdot e^{-\tilde{\beta}^{\prime}(x, y) \cdot \frac{\ln \left(\frac{\tilde{A}^{c} \cdot\left(\tilde{I} \tilde{I}^{c}(x, y)-\tilde{A}^{c}\right)}{\left(-\tilde{\beta}^{\prime}(x, y)(x, y)-\tilde{\beta}^{\prime}(x, y)\right)}\right)}{\left(c^{c}\right)}}}
$$

Let matrices $\quad M^{c}(x, y)=\ln \left(\frac{\check{A}^{c} \cdot\left(\tilde{I}^{c}(x, y)-\tilde{A}^{c}\right)}{\tilde{A}^{c} \cdot\left(\check{I}^{c}(x, y)-\check{A}^{c}\right)}\right), \quad N(x, y)=$ $\frac{-\tilde{\beta}^{\prime}(x, y)}{-\tilde{\beta}^{\prime}(x, y)+\tilde{\beta}^{\prime}(x, y)}$, the Eq. (6) can be rewritten as

$$
\rho^{c}=1+\frac{\tilde{I}^{c}-\tilde{A}^{c}}{\tilde{A}^{c} \cdot e^{\mathrm{M}^{c} \cdot \mathrm{N}}}
$$

Now that, our goal is to estimate the matrix $N$ since $\tilde{A}^{\mathrm{c}}$ and $\breve{A}^{\mathrm{c}}$ can be accurately obtained by [20]. Using the Eq. (3) and the redefined scattering maps, we have

$$
\left\{\begin{array}{l}
\tilde{\beta}^{\prime}(x, y)=\frac{-\log \left(\frac{\tilde{A}^{c}-\tilde{A}^{c}(x, y)}{\bar{A}^{c}\left(1-\rho^{c}(x, y)\right)}\right)}{d(x, y)} \\
\check{\beta}^{\prime}(x, y)=\frac{-\log \left(\frac{\check{\check{C}}^{c}-\check{I}^{c}(x, y)}{\tilde{A}^{c} \cdot\left(1-\rho^{c}(x, y)\right)}\right)}{d(x, y)}
\end{array}\right.
$$

Assuming the depth map $d$ is determined and $\rho^{c}=0$, we can get the upper boundary constraint of the scattering map through Eq. (8), that is

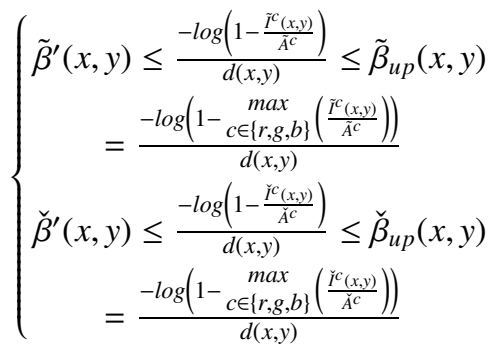

Note that, the matrix $N$ is just a ratio function in terms of scattering map $\tilde{\beta}^{\prime}$ and $\check{\beta}^{\prime}$. Consequently, we might as well regard the corresponding upper boundary constraint $\tilde{\beta}_{u p}$ and $\check{\beta}_{u p}$ as the rough "alternative" of scattering map $\tilde{\beta}^{\prime}$ and $\check{\beta}^{\prime}$, and therefore the $N$ can be approximately expressed as

$$
N(x, y) \approx \frac{-\tilde{\beta}_{u p}(x, y)}{-\tilde{\beta}_{u p}(x, y)+\check{\beta}_{u p}(x, y)}
$$

Considering that the atmospheric suspended particles obey the uniform distribution approximately within the local small-scale patch, which means the corresponding matrix $N$ shares the same property. Thus, we employ guided total variation model [21] to blur the $N$

$$
E(\hat{N})=\operatorname{argmin}\left(\|\hat{N}-N\|_{2}^{2}+\|\nabla \hat{N}-\nabla N\|_{2}^{2}+\|\nabla \hat{N}\|_{2}^{2}\right)
$$

where $\hat{N}$ is the blurred result of $N$. Finally, we substitute
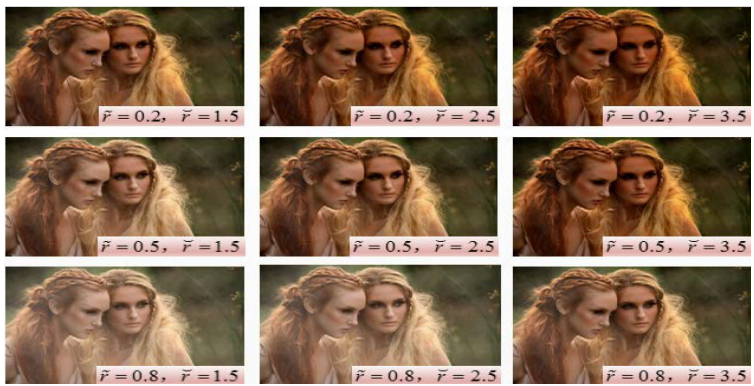

Fig. 1 The restoration results using different gamma correction factors. 
the estimated $\tilde{A}^{c}, M^{c}$ and $\hat{N}$ into Eq. (7), and restore the initial scene albedo $\rho_{1}^{c}$. However, the matrix $\hat{N}$ is not completely reliable because scene albedo is assumed to be zero when we compute the upper boundary, and it may lead to the over-saturated effect on the restored image. To compensate this limitation, the iteration-feedback strategy (initial

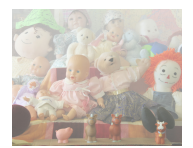

Hazy Image

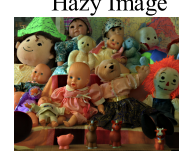

$j=3$

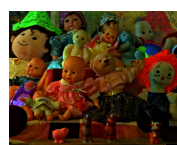

$j=1$

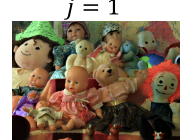

$j=4$
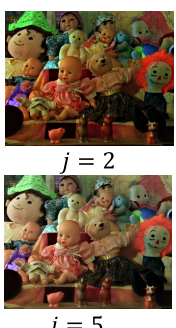

$j=5$
Fig. 2 Intermediate results of the iterative procedure.

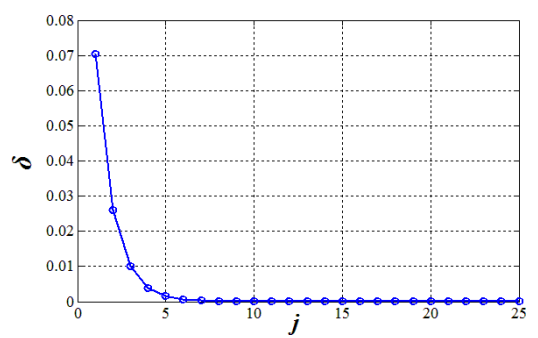

Fig. 3 Convergence curve of the proposed technique. iteration number $j$ is set to be 1) is designed and it can be detailed as:

Step1 Substitute the $j$ th scene albedo $\rho_{j}^{c}, \tilde{A}^{c}$ and $M^{c}$ into Eq. (7), and acquire the new matrix $\hat{N}_{j+1}$.

Step2 Using the $\hat{N}_{j+1}, \tilde{A}^{c}$ and $M^{c}$, recover the more visual compelling scene albedo $\rho_{j+1}^{c}$ via Eq. (7).

Step3 When the $\delta=\sum_{c \in\{r, g, b\}}\left\|\rho_{j+1}^{c}-\rho_{j}^{c}\right\|_{1} /$ res $\leq 0.003$ (res is image resolution) or the maximal iteration number $j_{\max }=25$ is satisfied, the iteration is terminated and the final result is set as $\rho_{\text {final }}^{c}=\rho_{j+1}^{c}$. Otherwise, update $j=j+1$ and return to Step 1.

Figure 1 gives the restoration results using different factors $\tilde{\gamma}$ and $\check{\gamma}$. As can be seen, with the decreasing $\tilde{\gamma}$ or the raising $\check{\gamma}$, haze can be removed more completely but the over-saturated phenomenon may also occur. In contrast, we select the $\tilde{\gamma}=0.5$ and $\tilde{\gamma}=2.5$ as our empirical parameters. Figure 2 further shows the intermediate results of the iterative procedure, and the corresponding convergence curve is shown in Fig. 3. From the curve, we find that actual iteration number is about 5 iterations.

\section{Experimental Comparison}

\subsection{Qualitative Comparison}

We test the proposed technique on various types of hazy images and compare with the physically-based dehazing approaches, including Meng et al.[22], He et al. [11], Zhu
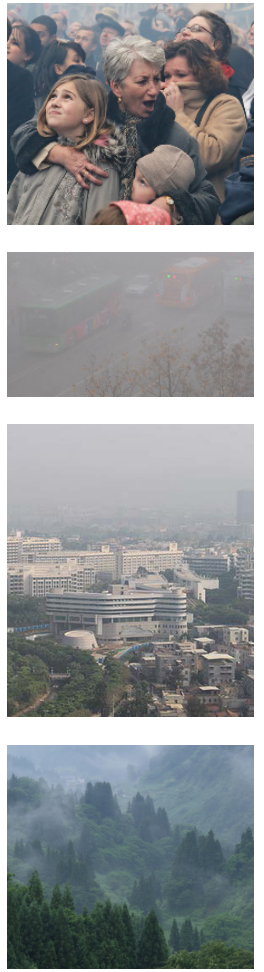
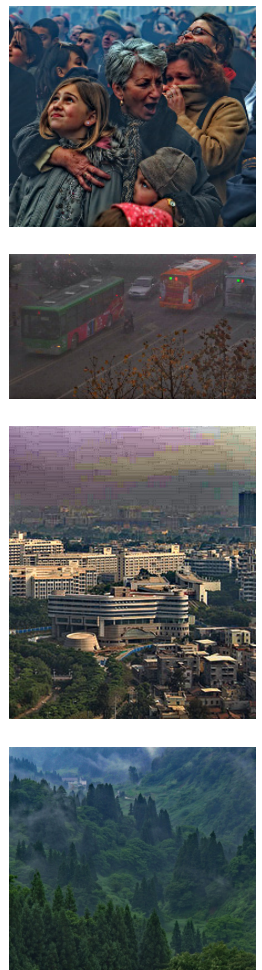
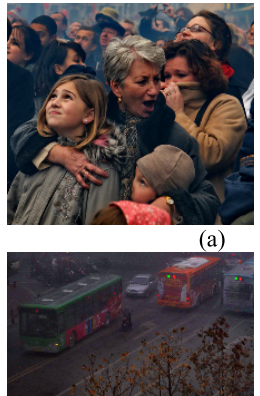

(b)

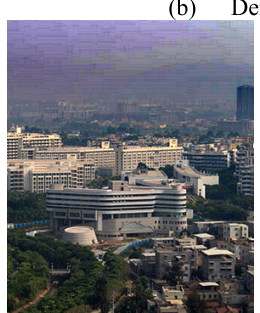

(c) Hazy Image with Sky region

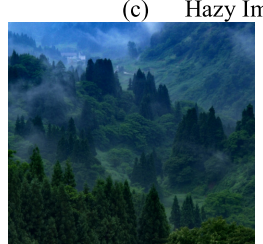

(d) Inhomogeneous Hazy Image
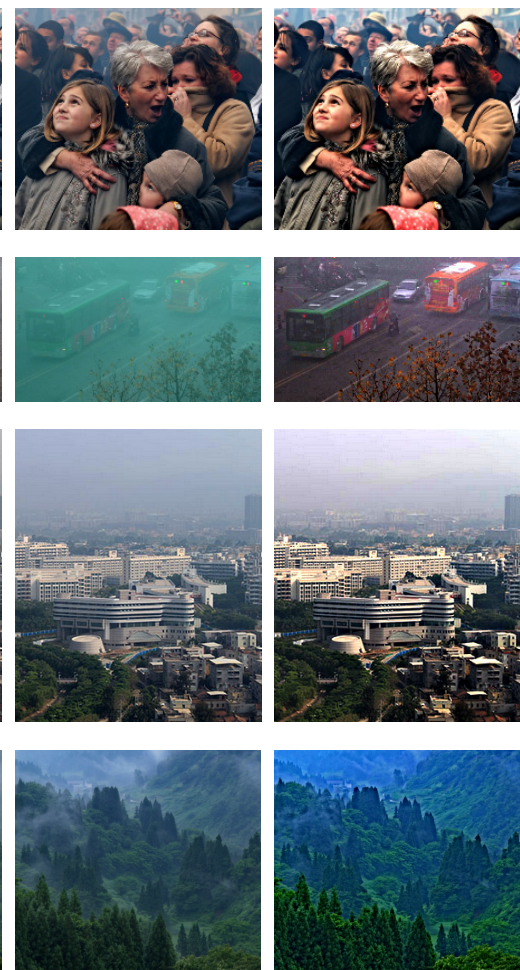

Fig. 4 From left to right: Hazy Images, Meng's results, He's results, Zhu's results, Cai's results and ours. 
Table 1 Quantitative comparison

\begin{tabular}{ccccc}
\hline Figures & techniques & $\bar{r}$ & FADE & $H$ \\
\hline \multirow{4}{*}{ Fig. 4(a) } & Meng et al & 1.3022 & 0.2074 & 0.3135 \\
\cline { 2 - 5 } & He et al & 1.0485 & 0.2444 & 0.0106 \\
\cline { 2 - 5 } & Zhu et al & 1.2415 & 0.3051 & 0.0133 \\
\cline { 2 - 5 } & Cai et al & 1.0784 & 0.2857 & 0.1462 \\
\cline { 2 - 5 } Fig. 4(b) & Our result & $\mathbf{1 . 3 9 6 7}$ & $\mathbf{0 . 1 7 6 2}$ & $\mathbf{0 . 0 0 8 6}$ \\
\cline { 2 - 5 } & Meng et al & 3.3706 & 0.8958 & 0.3012 \\
\cline { 2 - 5 } & He et al & 3.7257 & 0.6640 & 0.0223 \\
\cline { 2 - 5 } & Zhu et al & 1.8311 & 2.1971 & 0.0075 \\
\cline { 2 - 5 } & Cai et al & 1.6871 & 0.8467 & 0.4376 \\
\cline { 2 - 5 } Fig. 4(c) $)$ & Our result & $\mathbf{5 . 8 1 7 3}$ & $\mathbf{0 . 5 1 7 7}$ & $\mathbf{0 . 0 0 5 0}$ \\
\cline { 2 - 5 } & Meng et al & $\mathbf{2 . 4 8 6 4}$ & $\mathbf{0 . 2 4 3 6}$ & 0.0916 \\
\cline { 2 - 5 } & He et al & 2.1023 & 0.2864 & 0.0208 \\
\cline { 2 - 5 } & Zhu et al & 1.4608 & 0.5124 & 0.0107 \\
\hline \multirow{4}{*}{ Fig. 4(d) } & Cai et al & 1.3646 & 0.5185 & 0.0633 \\
\cline { 2 - 5 } & Our result & 1.8439 & 0.3225 & $\mathbf{0 . 0 0 6 5}$ \\
\cline { 2 - 5 } & Meng et al & 1.4097 & 0.4153 & 0.0374 \\
\cline { 2 - 5 } & He et al & 1.3840 & 0.2937 & 0.0037 \\
\cline { 2 - 5 } & Zhu et al & 1.2486 & 0.4513 & $\mathbf{0 . 0 0 0 5}$ \\
\hline & Cai et al & 1.1251 & 0.5752 & 0.0324 \\
\hline & Our result & $\mathbf{2 . 5 5 3 8}$ & $\mathbf{0 . 1 6 6 7}$ & 0.0021 \\
\hline
\end{tabular}

et al. [12], Cai et al. [14], as shown in Fig. 4. Through comparison, the restoration results of the proposed technique achieve higher visibility, better color fidelity, and with minimum blocking and halo artifacts, especially for the inhomogeneous hazy image.

\subsection{Quantitative Comparison}

In order to quantitative evaluation and rate the algorithms, we adopt several widely used indicators, including the mean ratio of the gradients at visible edges $\bar{r}$ [23], fog aware density evaluator FADE [5], and hue fidelity $H$ [24]. Generally, the higher values of $\bar{r}$ and the lower values of FADE, $H$ imply better dehazing results. Table 1 lists the quantitative analysis of the results in Fig. 4 in terms of the indicators mentioned. In some cases, it can be seen that the recovered results using our technique achieve the highest $\bar{r}$, the lowest FADE and $H$, which verify the advantage of the proposed method.

\section{Conclusion}

Different from the previous works, we provide a new clue for physically-based single image dehazing. Our strategy is to simulate two inputs taken in different weather conditions from one known hazy image using the gamma correction. Thus, the single image haze removal can be subtly converted into the multiple images haze removal, and the expected scene albedo can be effectively recovered on the basis of the invariance principle for scene structure and the inherent boundary constraint on the scattering function. Remarkably, we redefine the scattering coefficient as a variable with respect to the spatial location instead of a constant value, which could improve the restoration quality when processing inhomogeneous hazy image. However, the correction factors of the proposed method are determined empirically, thus we will seek the more reasonable factors that can achieve better restoration quality in our future work.

\section{References}

[1] K. Zuiderveld, Contrast Limited Adaptive Histogram Equalization, Academic Press, San Diego, 1994.

[2] C. Ancuti and C.O. Ancuti, "Effective Contrast-Based Dehazing for Robust Image Matching," IEEE Geosci. Remote Sens. Lett., vol.11, no.11, pp.1871-1875, Nov. 2014.

[3] Z. Mi, H. Zhou, Y. Zheng, and M. Wang, "Single image dehazing via multi-scale gradient domain contrast enhancement," IET Image Process., vol.10, no.3, pp.206-214, Nov. 2016.

[4] C.O. Ancuti and C. Ancuti, "Single Image Dehazing by Multi-Scale Fusion,” IEEE Trans. Image Process., vol.22, no.8, pp.3271-3282, May 2013.

[5] L.K. Choi, J. You, and A.C. Bovik, "Referenceless Prediction of Perceptual Fog Density and Perceptual Image Defogging," IEEE Trans. Image Process., vol.24, no.11, pp.3888-3901, July 2015.

[6] S.G. Narasimhan and S.K. Nayar, "Contrast restoration of weather degraded images," IEEE Trans. Pattern Anal. Mach. Intell., vol.25, no.6, pp.713-724, July 2003.

[7] R.T. Tan, "Visibility in bad weather from a single image," Proc. IEEE Conf. Comput. Vis. Pattern Recog., pp.1-8, 2008.

[8] K. Nishino, L. Kratz, and S. Lombardi, "Bayesian defogging," Int. J. Comput. Vision, vol.98, no.3, pp.263-278, July 2012.

[9] R. Fattal, "Single image dehazing," ACM Trans. Graphic., vol.27, no.3, pp.1-9, Aug. 2008.

[10] J.-P. Tarel and N. Hautiere, "Fast visibility restoration from a single color or gray level image," Proc. IEEE Int. Conf. Comput. Vis., pp.2201-2208, 2009.

[11] K. He, J. Sun, and X. Tang, "Single image haze removal using dark channel prior," IEEE Trans. Pattern Anal. Mach. Intell., vol.33, no.12, pp.2341-2353, Dec. 2011.

[12] Q. Zhu, J. Mai, and L. Shao, "A Fast Single Image Haze Removal Algorithm Using Color Attenuation Prior," IEEE Trans. Image Process., vol.24, no.11, pp.3522-3533, June 2015.

[13] K. Tang, J. Yang, and J. Wang, "Investigating Haze-Relevant Features in a Learning Framework for Image Dehazing," Proc. IEEE Conf. Comput. Vis. Pattern Recog., pp.2995-3002, 2014.

[14] B. Cai, X. Xu, K. Jia, C. Qing, and D. Tao, "DehazeNet: An Endto-End System for Single Image Haze Removal," IEEE Trans. Image Process., vol.25, no.11, pp.5187-5198, Jan. 2016.

[15] M. Ju, Z. Gu, and D. Zhang, "Single image haze removal based on the improved atmospheric scattering model," Neurocomputing, vol.260, pp.180-191, Oct. 2017.

[16] X. Ning, W. Li, and W. Liu, "A Fast Single Image Haze Removal Method Based on Human Retina Property," IEICE Trans. Inf. \& Syst., vol.E100-D, no.1, pp.211-214, 2017.

[17] H. Park, "An Improved Single Image Haze Removal Algorithm Using Image Segmentation," IEICE Trans. Inf. \& Syst., vol.E97-D, no.9, pp.2554-2558, 2014.

[18] K.B. Gibson, D.T. Võ, and T.Q. Nguyen, "An investigation of dehazing effects on image and video coding," IEEE Trans. Image Process., vol.21, no.2, pp.662-673, Feb. 2012.

[19] C. Liu, J. Zhao, Y. Shen, Y. Zhou, X. Wang, and Y. Ouyang, "Texture filtering based physically plausible image dehazing," The Visual Computer, vol.32, no.6-8, pp.911-920, 2016.

[20] N. Baig, M.M. Riaz, A. Ghafoor, and A.M. Siddiqui, "Image Dehazing Using Quadtree Decomposition and Entropy-Based Contextual Regularization," IEEE Signal Process. Lett., vol.23, no.6, pp.853-857, June 2016.

[21] M. Ju, D. Zhang, and X. Wang, "Single image dehazing via an improved atmospheric scattering model," Visual Comput., pp.1-13, Sept. 2016.

[22] G. Meng, Y. Wang, J. Duan, S. Xiang, and C. Pan, "Efficient image dehazing with boundary constraint and contextual regularization," Proc. IEEE Int. Conf. Comput. Vis., pp.617-624, 2013. 
[23] N. Hautière, J.-P. Tarel, D. Aubert, and É. Dumont, "Blind Contrast Enhancement Assessment by Gradient Ratioing at Visible Edges," Image Anal. Stereol., vol.27, no.2, pp.87-95, June 2008.
[24] D.J. Jobson, Z. Rahman, and G.A. Woodell, "Statistics of visual representation,” Proc. SPIE Int. Soc. Opt. Eng., pp.25-35, 2002. 